\title{
Cosmic radiation environment modelling for the ESEO mission
}

\author{
Boglárka Erdős, Attila Hirn, Balázs Zábori \\ Space Dosimetry Research Group \\ Hungarian Academy of Sciences Centre for Energy Research \\ Budapest, Hungary \\ erdos.boglarka@energia.mta.hu
}

\begin{abstract}
The development of the European Student Earth Orbiter (ESEO) was announced by the European Space Agency Education Office for students interested in the space exploration. The ESEO-TRITEL Team, supported by the Centre for Energy Research, Hungarian Academy of Sciences, joined this international cooperation by the development of the ESEO satellite version of the TRITEL 3D silicon detector telescope. Previous version of the TRITEL detector has been already operated successfully on board the European Columbus module of the International Space Station (ISS) and another version was installed in the Russian segment of the ISS as well. In the ESEOTRITEL experiment the anisotropies in the radiation field, the effects of the Earth shadow and the South Atlantic Anomaly (SAA) will be analyzed. The results will be compared with the fluxes calculated with the new AP-9 and AE-9 trapped proton and electron models, and possibly also compared against the previous AP-8 and AE-8 models for exploring differences. In this work the space radiation environment was reconstructed for the ESEO mission with the different models and the new results were compared to the ones obtained from the older model.
\end{abstract}

Keywords- ESEO; TRITEL; trapped particle radiation; dosimetry

\section{INTRODUCTION}

The European Student Earth Orbiter (ESEO) project was announced by the European Space Agency (ESA) in 2008 for students to acquire hands-on experience on real space project. Three Hungarian students' groups joined the mission to develop a subsystem and two payloads to the satellite, including the ESEO- TRITEL team. The development of the TRITEL threedimensional silicon detector telescope began in the Hungarian Academy of Sciences Centre for Energy Research several years ago. Absorbed dose, LET spectra in three directions, average quality factor of cosmic radiation and dose equivalent can be determined from the measured spectra [1]. After years of hard work ESEO was launched on 3 December 2018, but unfortunately most of the payloads, like the TRITEL detector, have not been switched on ever since. The planned mission time was 6 months with the possibility of extending it to one more year. Important orbital parameters of ESEO can be found in Table 1.

Since the original environmental modelling has been outdated by now, a new study was made to determine the trapped particle environment for a hypothetical mission using the older AP8/AE8 and the new AP9/AE9 models.

TABLE I. ESEO ORBITAL PARAMETERS

\begin{tabular}{|l|l|}
\hline Orbit type & Sun synchronous, circular \\
\hline Altitude & $575 \mathrm{~km}$ \\
\hline Inclination & $97.5^{\circ}$ \\
\hline LTDN & $10: 30$ \\
\hline
\end{tabular}

\section{TRAPPED ENERGETIC PARTICLE MODELS}

For more than two decades the AP8/AE8 trapped particle models $[2,3]$ were the standards for spacecraft design, and still they are the widely accepted models. These, however cannot benefit from the tremendous data that has been collected over the years, they only depend on two static maps measured decades ago. They also lack probability distribution and correct statistics and it has been proven that they over and underpredict measurements [4]. That is why the need for a new model arose. AP9/AE9 trapped particle models [5] use almost every available data collected since the $70 \mathrm{~s}$, of course omitting the data needed for the benchmark. They provide both measurement and gap-filling errors and also account for dynamic variations of space weather processes. They were also made to easily incorporate future datasets for improvement, such as datasets from the Van Allen Probes [5]. With the former models, two types of calculation could be made at solar maximum or at solar minimum. A major difference is that there is no solar-cycle dependence in AP9/AE9. It was left out because solar activity is highly unpredictable and for longer missions where solar cycle driven space weather effects become important, dynamical variations are statistically included in the model.

AP9/AE9 can be run in three modes: (1) mean mode, which only computes mean flux without uncertainties; (2) perturbed mean mode, which uses a random perturbation, consistent to gap-filling and measurement errors; and (3) the Monte Carlo mode, which uses autoregressive time-evolution model to get perturbed uncertainties and also an estimate of the dynamic variations due to space weather processes. These modes can be run resulting in mean average values or in user defined percentile levels. 


\section{TRAPPED PARTICLES ENVIRONMENT FOR ESEO}

In the following section the AP8/AE8 at solar minimum and maximum; AP9/AE9 mean, Monte Carlo mean and Monte Carlo $5^{\text {th }}$ and $95^{\text {th }}$ percentile models are compared to each other. Since AP8/AE8 models calculate mean fluxes, it can be compared only to the mean fluxes, but the $5^{\text {th }}$ and $95^{\text {th }}$ percentile fluxes are also shown to indicate uncertainties. All fluxes were calculated with ESA's SPace ENVironment Information System called SPENVIS [6]. For AP8/AE8 the threshold flux for exposure was $0.1 \mathrm{~cm}^{-2} \mathrm{~s}^{-1}$. To model the worst possible cases with AP8/AE8 for protons solar minimum, for electrons solar maximum condition was considered [7].

Figure 1 compares the orbit averaged (a) integral and (b) differential proton fluxes using the different trapped particle models for ESEO for a hypothetical mission starting from the 1 st September 2019 for 0.5 years. This mission time and length
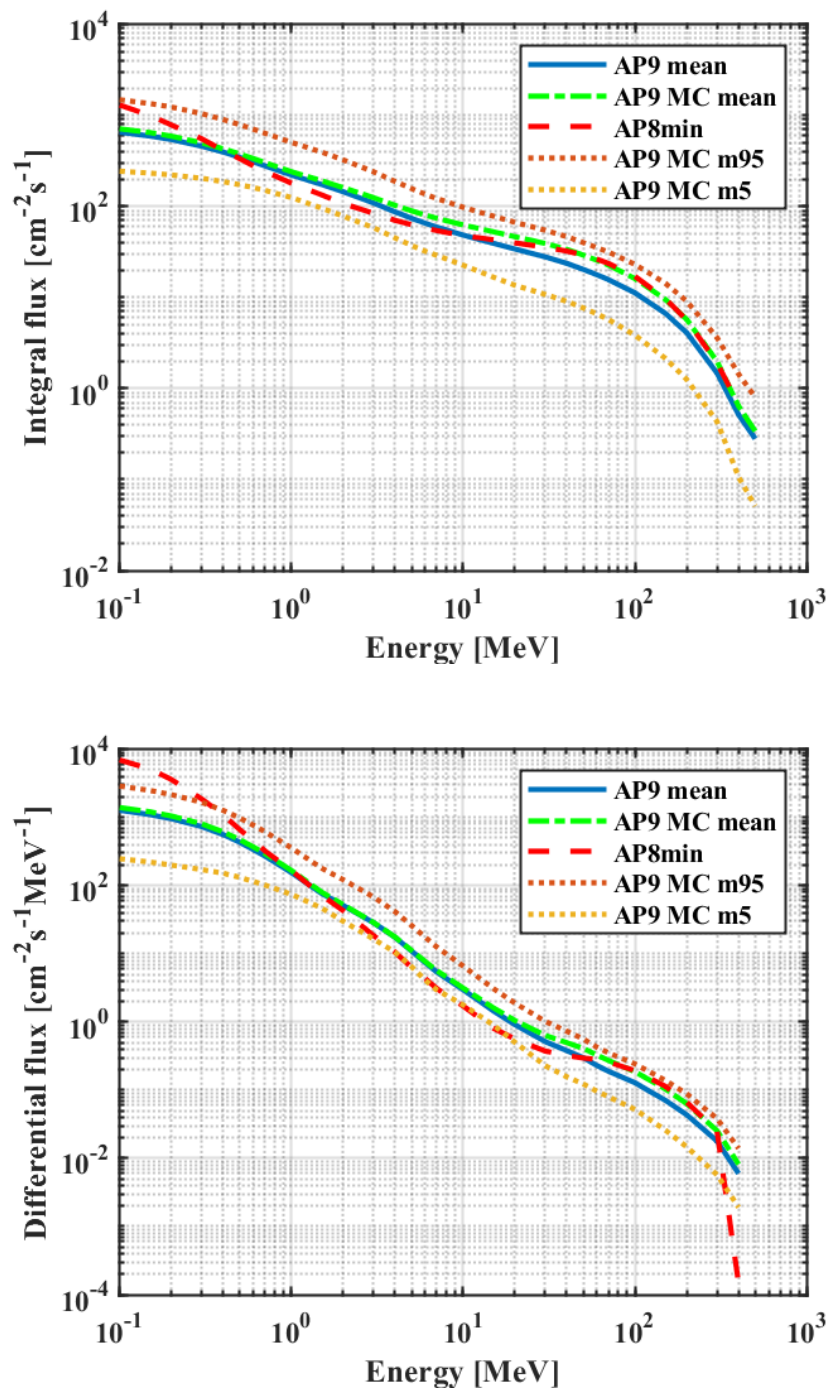

Fig. 1. Orbit averaged (a) integral and (b) differential proton fluxes for ESEO using the AP9 and the AP8 models for a hypotethical mission starting from the 1st September 2019 for 0.5 years. was chosen to model a future switching on of the TRITEL telescope. It shows that AP8min overestimates fluxes under $1 \mathrm{MeV}$ and over $50 \mathrm{MeV}$ but underestimates in between these regions compared to AP9. TRITEL would measure between $18 \mathrm{MeV}$ and $10 \mathrm{GeV}$ [8], mostly where the fluxes were underestimated. Higher fluxes mean slightly higher count rates in the region where only around 720 counts per seconds are expected at maximum with the current $1.2 \mathrm{~mm}$ aluminum shielding [9]. Compared to this, the maximum count rate of TRITEL is $50000 \mathrm{cps}$. It means that slightly higher count rates could be expected in the main region, which improves statistics. Also, slightly thinner shielding could have been chosen to get similar results. However, $1.2 \mathrm{~mm}$ was also chosen to be equivalent to the lower limit of the effective thickness of a typical spacesuit. The figure also shows that AP9 mean and AP9 Monte Carlo mean is similar to each other, but the Monte Carlo mode models predict higher fluxes above around
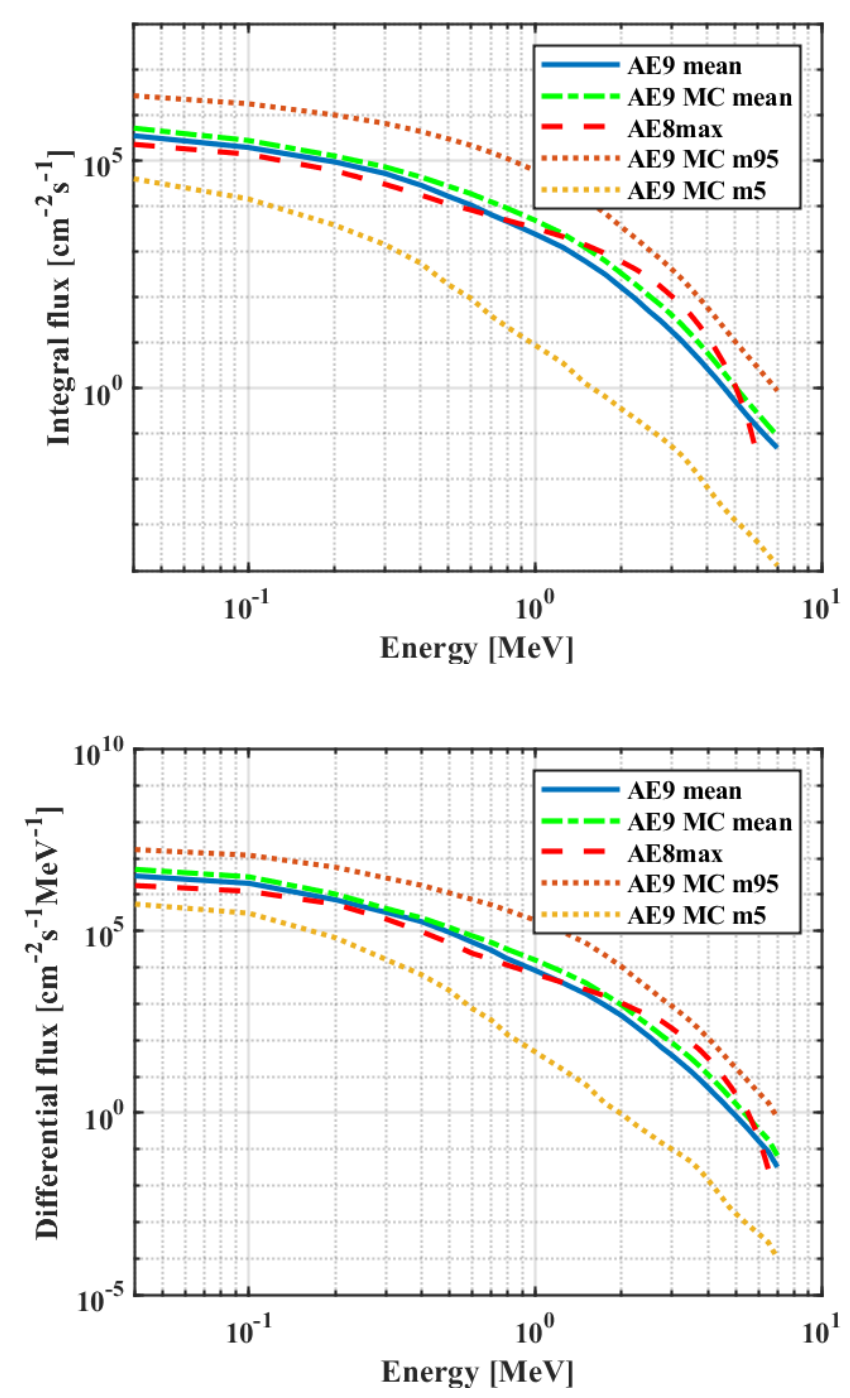

Fig. 2. Orbit averaged (a) integral and (b) differential electron fluxes for ESEO using the AE9 and the AE8 models for a hypotethical mission starting from the 1 st September 2019 for 0.5 years. 
$10 \mathrm{MeV}$, making the results there more similar to AP8. However, AP8 cuts off earlier than the new models.

Figure 2 compares the same data but for electrons. AE8 orbit averaged flux is similar to the new model at low energies, but overpredicts above $1 \mathrm{MeV}$, right in the measuring range of TRITEL and cuts off earlier as well. This could mean smaller real count rates for electrons.

For the next two sets of figures, all data was modelled with AE9/AP9/SPM V1.50.001 package developed and distributed by NASA. Figure 3 shows trapped proton integral flux at $2 \mathrm{MeV}$ along the orbit for one day, $1^{\text {st }}$ September 2019 as a function of geographical longitude and latitude. One square corresponds to 60 seconds. Nonzero flux values contour the South Atlantic Anomaly (SAA). The shape of SAA is similar, but the overall extent of the shape is bigger for AP9. Although AP8min has higher maximum, the peak of SAA is smaller and at a different position. Since in the model the peak is larger and it takes approximately 12 minutes to go over it, it would improve the resolution if reading would be around 6 minutes instead of the currently used 10 minutes. If the satellite passing
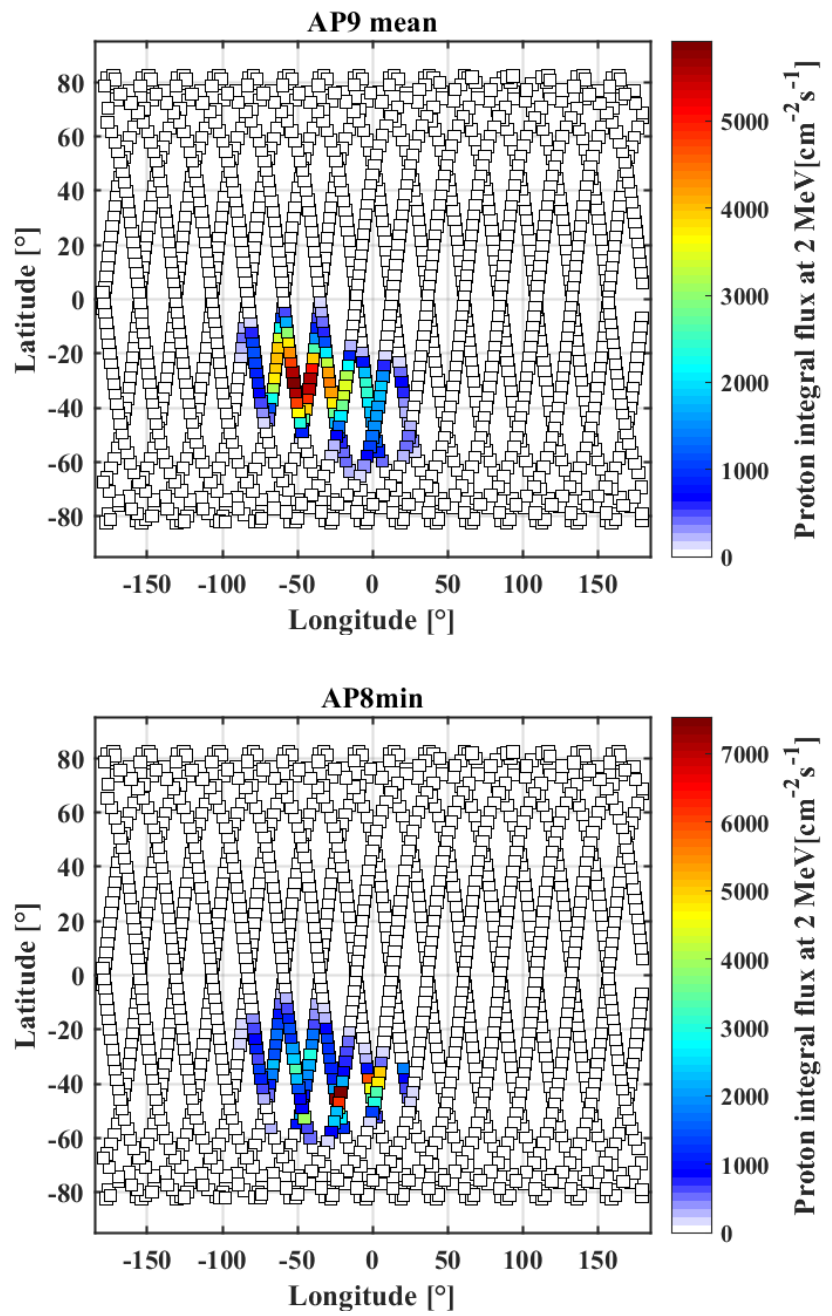

Fig. 3. Proton integral flux at $2 \mathrm{MeV}$ with (a) AP9 mean and (b) AP8min as a function of geographical longitude and latitude. Flux was calculated for only one day, $1^{\text {st }}$ September 2019. only at the SAA very edge, it only takes about 4 minutes, which would be measured not accurate enough with 10 minutes of reading time.

Figure 4 shows trapped electron integral flux at $0.1 \mathrm{MeV}$ along the orbit for one day, $1^{\text {st }}$ September 2019 as a function of geographical longitude and latitude. One square corresponds to 60 seconds. Nonzero flux values contour the SAA and polar regions. Similar to proton fluxes, AE9 calculates a larger dimension for SAA, but less electron flux for the polar regions. This also means that shorter reading time would improve data resolution in these regions. The maximum of the flux is almost equal in both cases, the main difference is the shape of regions of interest.

\section{SUMMARY}

Within the framework of this study, the differences between the widely used older AP8/AE8 and newly developed AP9/AE9 trapped energetic particles models were investigated. It was found that there are smaller differences in the orbit averaged
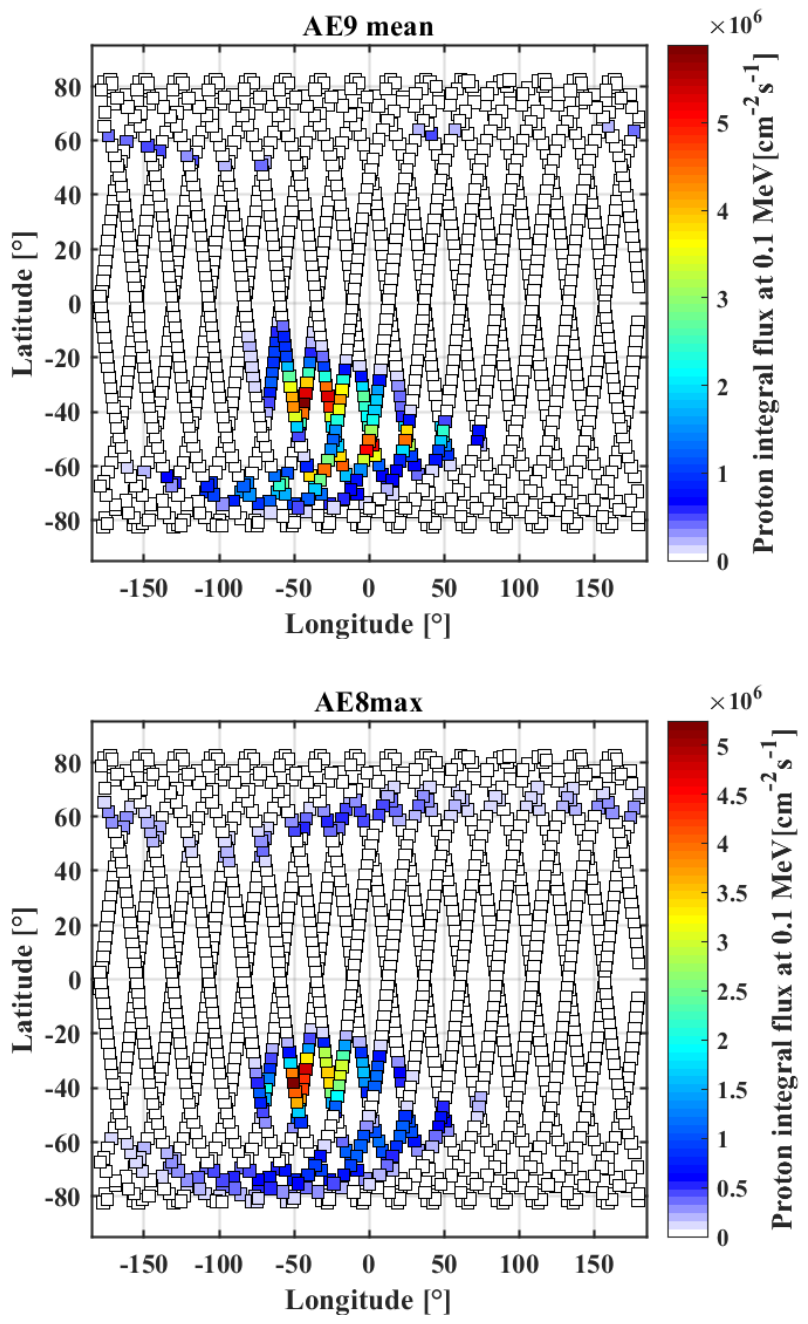

Fig. 4. Electron integral flux at $0.1 \mathrm{MeV}$ with (a) AP9 mean and (b) AP8min as a function of geographical longitude and latitude. Flux was calculated for only one day, $1^{\text {st }}$ September 2019. 
fluxes. AP8/AE8 under and overestimates AP9/AE9 in regions. Also, the extent of the SAA is different; if the integral flux along the orbit was examined at a given energy. Based on these, shorter spectrum acquisition time is recommended in the future, to have a reasonably accurate resolution of the data measured in SAA, to be able to compare models with data. It has been previously shown, that AE9 under and overpredicts quantities around SAA, since it lacks proper electron data near that region [5]. However, the developers are aware of these shortcomings and the model will be improved with future measurements.

The aim of this work was to study the effects of the new model on detector development through the example of ESEO. If our instrument can be turned on in the future on ESEO, the difference could be studied not only in theory but through measurements as well and would be helpful designing similar detectors telescopes in the future.

\section{ACKNOWLEDGMENTS}

The authors acknowledge the services provided by the organizers of the ESEO project, especially the European Space Agency Educational Office, SITAEL, and the experts of the organizations who supported the student teams throughout the project.

Phase D2/E of the ESEO-TRITEL Scientific Payload is funded in the frame of the ESA contract No. 4000124167.

The authors wish to thank the work of the ESEO-TRITEL team members and the scientific and engineering support provided by the experts of the MTA Centre for Energy Research.

\section{REFERENCES}

[1] T. Pázmándi, S. Deme, E. Láng, "Space dosimetry with the application of a 3D silicon detector telescope: response function and inverse algorithm", Radiat. Prot. Dosimetry, Volume 120, pp. 401-404, 2006.

[2] D. M. Sawyer, J. I. Vette, "AP-8 trapped proton environment for solar maximum and solar minimum", NSSDC/WDC-A-R\&S 76-06, 1976.

[3] J. I. Vette, "The AE-8 trapped electron model environment, NSSDC/WDC-A-R\&S 91-24, 1991.

[4] J.-M. Lauenstein ; J.L. Barth, "Radiation belt modeling for spacecraft design: model comparisons for common orbits", IEEE Radiation Effects Data Workshop. 102 - 109. 10.1109/REDW.2005.1532674, 2005.

[5] G. P. Ginet, T. P. O'Brien, S. L. Huston, W. R. Johnston, et al., "AE9, AP9 and SPM: New models for specifying the trapped energetic particle and space plasma environment" Space Science Reviews, Volume 179, Issue 1-4, pp 579-615, November 2013.

[6] D. Heynderickx, B. Quaghebeur, E. Speelman and E. Daly, "ESA's Space Environment Information System (SPENVIS) - A WWW interface to models of the space environment and its effects", AIAA-2000-0371, 2000 .

[7] D. Heynderickx, "Radiation belt modelling in the framework of space weather effects and forecasting", J. Atmos. Sol.-Terr. Phys, Volume 64, pp. 1687-1700, 2000.

[8] B. Zábori, A. Hirn, P. Bencze, "The relationship between plasma effects and cosmic radiation studied with TriTel-LMP measurements during the ESEO mission”, J. Adv. Space Res., Volume 48, pp 240-253, 2011.

[9] B. Zábori, A. Hirn,'TriTel 3 dimensional space dosimetric telescope in the European Earth Orbiter project of ESA", Acta Astronautica, Volume 71, pp 20-31, 2012. 